\title{
Molecular imaging of oxidized collagen quantifies pulmonary and hepatic fibrogenesis
}

\author{
Howard H. Chen, ${ }^{1}$ Philip A. Waghorn, ${ }^{1}$ Lan Wei, ${ }^{2}$ Luis F. Tapias, ${ }^{3}$ Daniel T. Schühle, ${ }^{1}$ \\ Nicholas J. Rotile, ${ }^{1}$ Chloe M. Jones, ${ }^{1}$ Richard J. Looby, ${ }^{1}$ Gaofeng Zhao, ${ }^{3}$ Justin M. Elliott, ${ }^{3}$ \\ Clemens K. Probst, ${ }^{4}$ Mari Mino-Kenudson, ${ }^{5}$ Gregory Y. Lauwers, ${ }^{5}$ Andrew M. Tager, ${ }^{4}$ \\ Kenneth K. Tanabe, ${ }^{2}$ Michael Lanuti, ${ }^{3}$ Bryan C. Fuchs, ${ }^{2}$ and Peter Caravan ${ }^{1}$
}

'Athinoula A. Martinos Center for Biomedical Imaging and the Institute for Innovation in Imaging, Department of Radiology, ${ }^{2}$ Division of Surgical Oncology, Massachusetts General Hospital Cancer Center, ${ }^{3}$ Division of Thoracic Surgery, ${ }^{4}$ Division of Pulmonary and Critical Care Medicine and the Center for Immunology and Inflammatory Diseases, ${ }^{5}$ Department of Pathology, Massachusetts General Hospital and Harvard Medical School, Boston, Massachusetts, USA.

Fibrosis results from the dysregulation of tissue repair mechanisms affecting major organ systems, leading to chronic extracellular matrix buildup, and progressive, often fatal, organ failure. Current diagnosis relies on invasive biopsies. Noninvasive methods today cannot distinguish actively progressive fibrogenesis from stable scar, and thus are insensitive for monitoring disease activity or therapeutic responses. Collagen oxidation is a universal signature of active fibrogenesis that precedes collagen crosslinking. Biochemically targeting oxidized lysine residues formed by the action of lysyl oxidase on collagen with a small-molecule gadolinium chelate enables targeted molecular magnetic resonance imaging. This noninvasive direct biochemical elucidation of the fibrotic microenvironment specifically and robustly detected and staged pulmonary and hepatic fibrosis progression, and monitored therapeutic response in animal models. Furthermore, this paradigm is translatable and generally applicable to diverse fibroproliferative disorders.

Authorship note: H.H. Chen and P.A. Waghorn contributed equally to this work.

Conflict of interest: P. Caravan has equity in and is a consultant to Collagen Medical LLC, has equity in Reveal Pharmaceuticals Inc, and has research support from Biogen, Pfizer, and Agilent. This work was not supported by any of these companies. A patent has been filed (application number, W02015/085005) by P. Caravan and Massachusetts Ceneral Hospital on the molecular probe described here. B.C. Fuchs is a consultant to Collagen Medical LLC.

Submitted: October 28, 2016

Accepted: April 25, 2017

Published: June 2, 2017

Reference information: JCI Insight. 2017;2(11):e91506. https:// doi.org/10.1172/jci.insight.91506.

\section{Introduction}

Fibrosis is the accumulation of extracellular matrix molecules that make up scar tissue as a consequence of chronic tissue injury $(1,2)$. Pulmonary fibrosis $(3,4)$, hepatic cirrhosis (5), atrial fibrillation (6), myocardial infarction (7), hypertrophic cardiomyopathy (8), and diabetic nephropathy (9) are among the more common diseases characterized by tissue fibrosis (1), which together account for nearly half of the deaths in the industrialized world and represent an enormous unmet medical challenge $(10,11)$. The management of patients with fibrotic diseases is complicated by the absence of noninvasive methods to assess disease activity that could predict fibrosis progression and disease course in individual patients, and provide early assessments of patient responses to novel therapies now being developed $(12,13)$. Invasive biopsy can provide some measures of disease activity, but patient compliance and the increased risk of complications renders repeated biopsies to evaluate disease progression or response to treatment a suboptimal strategy. There is thus a need for assessing disease activity noninvasively.

The recognition that biochemical features of fibrosis are conserved across tissue and organ types has led to the hope that universal targets exist for diverse fibrotic diseases $(14,15)$. During fibrosis, type-I collagen is overexpressed in sufficient quantity to be detected and quantified by a targeted molecular magnetic resonance imaging (MRI) peptide probe (16-20). However, this approach cannot distinguish active fibrogenesis because of slow collagen turnover in tissue. Preceding collagen crosslinking, lysyl oxidase (LOX) and LOX-like enzymes are upregulated and oxidize the collagen lysine $\varepsilon$-amino groups to aldehydes (allysine) (21-23). Allysine is abundant during fibrogenesis owing to the high substrate concentration and catalytic activity of LOXs. Collagen is present at more than $10 \mu \mathrm{M}$ in fibrotic tissue and there are more than 100 lysine residues per collagen monomer (24). When fibrogenesis subsides, LOX activity ceases and the reactive allysine residues are consumed by condensation reactions. We hypothesize that an allysine-reactive MRI contrast agent that targets the catalytic lysine oxidation process can stage disease progression and monitor treatment response in multiple fibrotic disease organs. 


\section{Results}

To target oxidized collagen, we designed the probe Gd-Hyd based on a Gd-DOTA chelate conjugated to a hydrazide moiety (Figure 1A and Supplemental Figure 1; supplemental material available online with this article; https://doi.org/10.1172/jci.insight.91506DS1). The hydrazide undergoes a reversible condensation reaction with aldehydes to yield a hydrazone. Gd-DOTA is chosen for its stability, inertness, and safety profile $(25,26)$. We sought a very hydrophilic, anionic MR-sensitive compound that minimizes nonspecific protein binding, ensures an extracellular distribution, and promotes rapid renal elimination, thus reducing background signal and limiting toxicity. As a negative control we synthesized Gd-DiMe (Figure 1A and Supplemental Figure 2), the dimethyl hydrazide analog incapable of undergoing a condensation reaction with an aldehyde.

Gd-Hyd showed essentially no binding to bovine serum albumin (BSA), but did bind reversibly to BSA that had been oxidized to generate $16 \mathrm{nmol}$ of aldehydes per mg of protein (Figure 1B). Addition of cyanoborohydride to the reaction then resulted in irreversible binding of Gd-Hyd to the protein, as expected for reduction of the hydrazone to an irreversible hydrazide linkage. Gd-DiMe showed no affinity for BSA or oxidized BSA. The relaxivity, a measure of MR efficiency, was $4.07 \mathrm{mM}^{-1} \mathrm{~s}^{-1}$ for Gd-Hyd, increased by $12.3 \%$ to $4.57 \mathrm{mM}^{-1} \mathrm{~s}^{-1}$ in the presence of oxidized BSA, where the bound species showed a robust $238 \%$ increase $\left(13.74 \mathrm{mM}^{-1} \mathrm{~s}^{-1}\right)$ in relaxivity after removal of the unbound Gd-Hyd probe by ultrafiltration (Figure $1 \mathrm{C}$ and Supplemental Figures 3 and 4). Gd-DiMe showed negligible increase in relaxivity in the presence of BSA or oxidized BSA. A tissue with high LOX activity is the aorta, in which turnover of elastin and collagen is high. Gd-Hyd binds to porcine aorta with a $\mathrm{K}_{d}$ of $650 \pm 61 \mu \mathrm{M}$, while Gd-DiMe showed no affinity (Figure 1D). Analysis of allysine in aorta revealed $7.50 \mu \mathrm{mol} / \mathrm{g}$ tissue, indicating that the target was present at levels sufficient for binding Gd-Hyd.

Using whole-body MRI, we observed immediate enhancement of the blood pool and clearance through the kidneys after Gd-Hyd administration. In normal mice, Gd-Hyd underwent rapid elimination from the blood ( $\mathrm{t}_{1 / 2}$ of $4.7 \pm 1.7$ minutes) into the urine. Apart from a first-pass blood pool effect, there was no signal increase in the liver or other organs. Gd-DiMe showed similar blood clearance ( $t_{1 / 2}$ of $4.2 \pm 1.8$ minutes). Whole-body biodistribution after Gd-Hyd injection indicated that more than $99.5 \%$ of the injected dose of gadolinium was eliminated from the body at 24 hours after injection (Supplemental Table 1).

We evaluated the ability of Gd-Hyd to detect pulmonary fibrosis in a bleomycin lung injury mouse model. Two weeks after bleomycin insult, MR images were acquired before and at 12 minutes after injection of either Gd-Hyd or Gd-DiMe (Figure 1E). Twenty-four hours later, imaging was repeated in the same mouse with the other probe to directly assess Gd-Hyd specificity. We measured the change in contrastto-noise ratio $(\triangle \mathrm{CNR})$ of lung signal relative to adjacent skeletal muscle before and after probe injection. $\triangle \mathrm{CNR}$ was higher in all of the 8 mice (3.8-fold higher on average) receiving Gd-Hyd compared with $\Delta \mathrm{CNR}$ measured after Gd-DiMe injection (Figure 1F). Gd-Hyd administration led to a 3.7-fold increase in $\Delta \mathrm{CNR}$ in bleomycin-injured mice compared with sham-treated mice. The difference in $\triangle \mathrm{CNR}$ between bleomycininjured and sham mice was not significant for Gd-DiMe (Figure 1G).

We then studied whether Gd-Hyd-enhanced MRI could not only monitor disease progression in the bleomycin model, but also monitor the response to treatment in bleomycin-injured mice treated with the pan-LOX inhibitor, $\beta$-aminopropionitrile (BAPN) (27). We imaged mice that had been administered bleomycin or PBS at 1 or 2 weeks after challenge, and bleomycin mice treated prophylactically with BAPN for 2 weeks. After imaging, the right lung was fixed and stained with Sirius Red (collagen/fibrosis stain) and with H\&E (Figure 2A). Slides were graded for pulmonary fibrosis using the Ashcroft system (28) by a pathologist blinded to the study (Figure 2B). At 1 week after bleomycin, the architecture of the lung was damaged, with a mean Ashcroft score of 4.1 (range 3-5) and progressed to 5.4 (range 0-7) by 2 weeks. The collagen proportional area (CPA) stained positive for Sirius Red (Figure 2C) was moderately elevated at 1 week (16.7\% vs. sham: 9.6\%), and worsened at 2 weeks (19.4\%). BAPN treatment had a modest, but positive effect on the lung architecture as assessed by $H \& E$ and Sirius Red staining, with significantly lowered Ashcroft score (2.2), and reduced CPA (14.4\%), although the reduction in CPA was not statistically significant.

The left lung was homogenized and assayed for hydroxyproline (HYP) content (Figure 2D), Lox family gene expression (Figure 2E and Supplemental Figure 7), total LOX activity (Figure 2F), and allysine content (Figure 2G). Consistent with CPA, HYP was not significantly increased until 2 weeks (106.5 $\mu \mathrm{g} /$ lung vs. sham: $48.6 \mu \mathrm{g} / \mathrm{lung}$ ), and was not significantly reduced by BAPN (105.7 $\mu \mathrm{g} / \mathrm{lung})$. mRNA levels of Loxl2 (Figure 2E) and Loxl3 (Supplemental Figure 7) were both significantly elevated during disease 

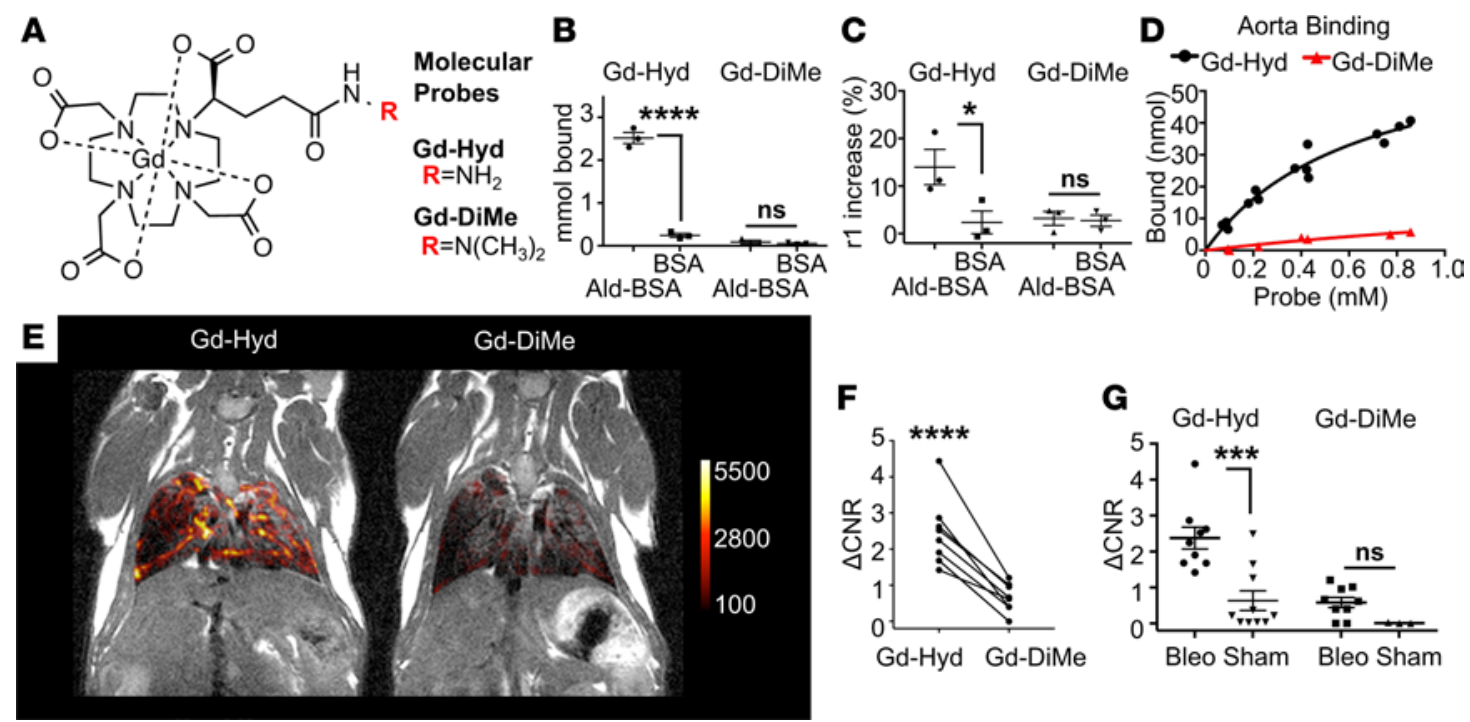

Figure 1. Gd-Hyd specifically detects peptidyl aldehydes, in oxidized albumin and porcine tissue in vitro, and in bleomycin-injured mouse lungs in vivo. (A) Structures of Gd-Hyd (active probe) and Gd-DiMe (inactive control). Gd-Hyd bound to allysine in oxidized bovine serum albumin (Ald-BSA) but not to unmodified BSA, as assessed by direct protein binding $(\mathbf{B}, n=3)$ and by relaxivity increase $(\mathbf{C}, n=3)$. Gd-DiMe showed no binding to either construct. (D) Gd-Hyd, but not Gd-DiMe, bound to allysine-rich porcine aorta. (E) Consecutive coronal MR images, taken 24 hours apart, show strong lung enhancement (false colored) by Gd-Hyd but not Gd-DiMe in the same mouse 2 weeks after bleomycin lung injury. (F) Sequential MRI of bleomycin-injured mice quantified by lung/muscle change in contrast-to-noise ratio ( $\triangle \mathrm{CNR})(n=8)$. (C) Gd-Hyd and Gd-DiMe $\Delta$ CNR in bleomycin-injured mice $(n=8)$ and sham controls $(n=10) .{ }^{*} P<0.05,{ }^{* * *} P<0.001,{ }^{* * *} P<0.0001$, ns, not significant; ANOVA.

progression. BAPN treatment did not reduce Lox family, $\alpha$-smooth muscle actin (Sma), or collagen type $1 \alpha 1$ (Col1a1) gene expression (Figure 2E and Supplemental Figure 8). Total LOX enzymatic activity in the fibrotic lung tissue was several fold higher (4.5-fold at 1 week; 3.4-fold at 2 weeks) than in sham animals (Figure 2F). BAPN treatment had a marked effect, significantly abolishing the LOX activity to levels similar to the sham-treated mice. While lysine oxidation is catalytically driven by LOX, the noncatalytic condensation reaction of the resultant aldehyde to create crosslinks is slow. We used an HPLC method to directly quantify the amount of allysine present in the diseased tissue. Allysine, a product of the collagen substrate and the total LOX enzymatic activity, was elevated at 1 week after bleomycin challenge, reached significantly increased levels at 2 weeks after bleomycin, and was significantly reduced by LOX inhibition with BAPN (Figure 2G). Unlike HYP, a common measure of total collagen, allysine (oxidized lysine) is a measure of oxidized collagen and the aldehyde target available for interaction with Gd-Hyd.

Gd-Hyd-enhanced MRI tracked disease progression and allysine tissue levels, quantifiable by the 4.3fold increase in $\triangle \mathrm{CNR}$ at 1 week, and 6.5-fold increase at 2 weeks after bleomycin relative to sham mice (Figure 2, H and I). BAPN treatment resulted in a robust reduction in Gd-Hyd-enhanced MRI signal where $\triangle \mathrm{CNR}$ was reduced to levels observed in sham animals. $\triangle \mathrm{CNR}$ quantification of MRI thus mirrored the changes in allysine concentration and LOX activity in the lungs of bleomycin-injured mice, and consequently is able to monitor disease progression and treatment response.

Next, we examined whether Gd-Hyd-enhanced MRI could distinguish active fibrogenesis from stable, mature fibrotic scar. At 14 days after bleomycin we showed that both LOX tissue activity and allysine concentration are significantly elevated. We hypothesized that at a later time point after bleomycin injury, fibrogenesis would slow or cease, but the fibrotic scar would remain. Here we used a lower dose of bleomycin (1 unit/kg compared with 2.5 units/ $\mathrm{kg}$ in the prior experiments) to limit mortality at later time points. We characterized disease progression in this model at 1 week, 2 weeks, and 4 weeks following bleomycin challenge with histology (Sirius Red staining for collagen, Verhoeff's elastin staining, and H\&E staining), and biochemical measures (LOX activity, HYP and allysine content); imaging was performed in the 2-week and 4-week cohorts along with animals that underwent a sham procedure with PBS.

In this model, disease progression was confirmed by histological (Figure 3A) and biochemical assessments. At 1 week after bleomycin, the architecture of the lung was damaged, with a mean Ashcroft score of 4.7 (range 4.5-5), progressed to 6.4 (range 5-7) by 2 weeks, and persisted (6.7, range 6-7.5) at 4 weeks. 

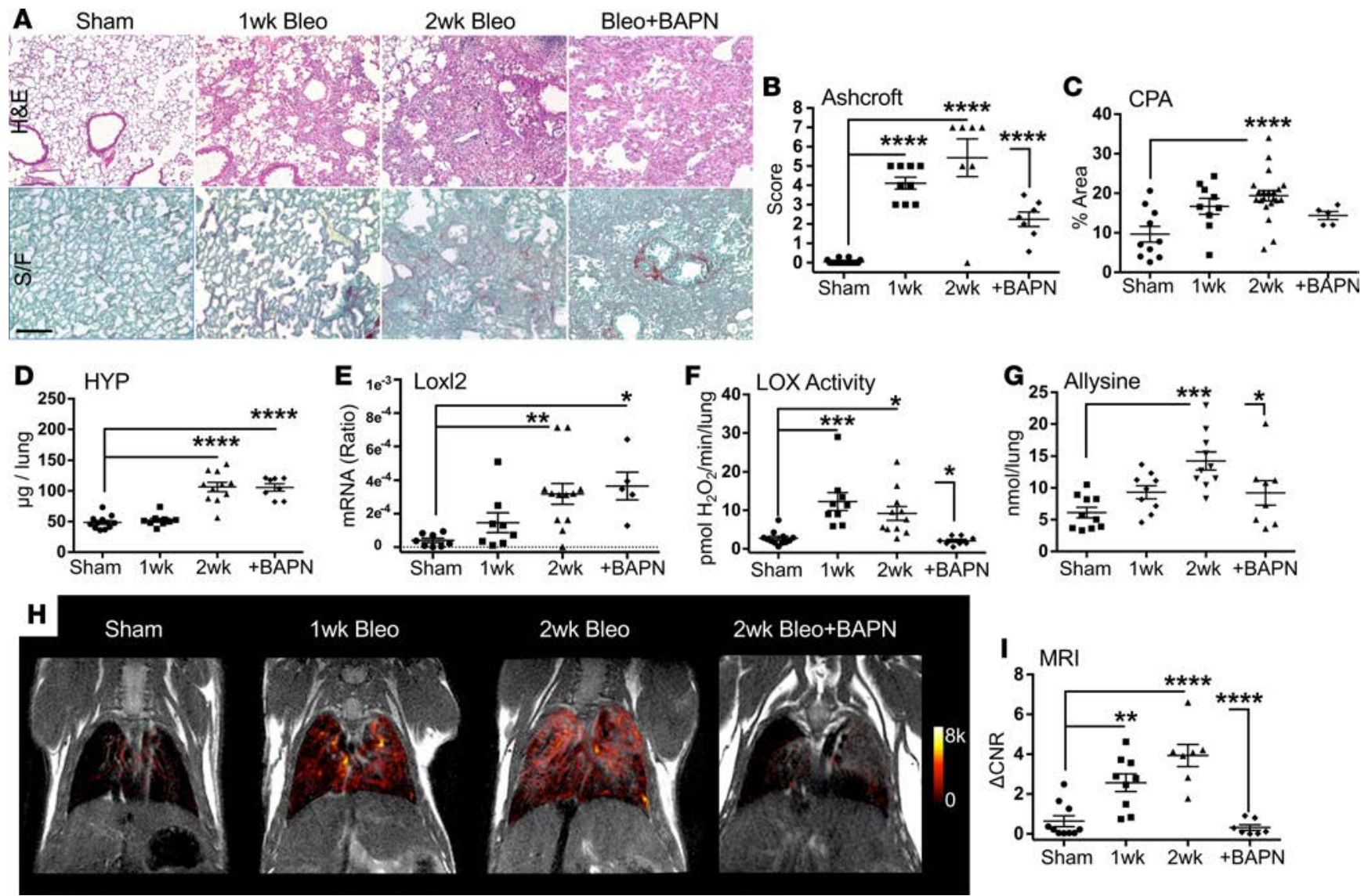

Figure 2. Gd-Hyd-enhanced MRI quantitatively tracks pulmonary fibrosis progression and detects response to lysyl oxidase (LOX) inhibition. (A) H\&E- and Sirius Red/fast green (S/F)-stained sections of lung tissues from mice that underwent a sham procedure (sham, $n=10$ ), were challenged with bleomycin intratracheally 1 week ( $1 w k, n=9$ ) or 2 weeks prior ( 2 wk, $n=17$ ), and 2-week bleomycin-challenged mice treated with pan-LOX inhibitor BAPN $(n=7)$. Lung injury as assessed by Ashcroft score (B), collagen proportional area (CPA) (C), lung hydroxyproline (HYP) content (D), LoxI2 expression (E), LOX activity (F), and allysine content for oxidized lung collagen (C). (H) Gd-Hyd-enhanced coronal MR images, and the change in contrast-to-noise ratio ( $\triangle \mathrm{CNR}$ ) quantification (I). Scale bar: $100 \mu \mathrm{m} .{ }^{*} P<0.05,{ }^{* *} P<0.01,{ }^{* *} P<0.001,{ }^{* * *} P<0.0001$; ANOVA.

The CPA (Figure 3B) was nonsignificantly elevated at 1 week $(8.4 \% \pm 2.9 \%$ vs. sham: $6.5 \% \pm 1.9 \%)$, but significantly increased at 2 weeks $(16.5 \% \pm 7.8 \%)$, and further increased at 4 weeks $(20.2 \% \pm 5.2 \%)$. The relative area stained positive for elastin (Supplemental Figure 9) was moderately elevated after 1 week $(15.1 \% \pm 4.0 \%$ vs. sham: $12.5 \% \pm 3.1 \%)$ and 2 weeks $(18.4 \% \pm 4.1 \%)$, but was significantly increased after 4 weeks $(30.4 \% \pm 8.4 \%)$. Consistent with CPA, HYP content (Figure 3C) was not significantly elevated at 1 week $(80.9 \pm 15.1 \mu \mathrm{g} /$ lung vs. sham: $51.5 \pm 4.5 \mu \mathrm{g} / \mathrm{lung})$, significantly increased at 2 weeks $(113.5 \pm$ $21.0 \mu \mathrm{g} / \mathrm{lung}$ ), and continued to increase at 4 weeks (144.8 $\pm 36.5 \mu \mathrm{g} / \mathrm{lung}$ ). LOX activity (Figure 3D) was significantly elevated at 1 week $\left(3.9 \pm 1.1\right.$ pmol $\mathrm{H}_{2} \mathrm{O}_{2} / \mathrm{min} / \mathrm{lung}$ vs. sham: $\left.1.6 \pm 0.5 \mathrm{pmol} \mathrm{H}_{2} \mathrm{O}_{2} / \mathrm{min} / \mathrm{lung}\right)$, further increased at 2 weeks ( $\left.10.9 \pm 1.4 \mathrm{pmol} \mathrm{H}_{2} \mathrm{O}_{2} / \mathrm{min} / \mathrm{lung}\right)$, but significantly decreased at 4 weeks (4.9 $\pm 1.3 \mathrm{pmol} \mathrm{H}_{2} \mathrm{O}_{2} / \mathrm{min} / \mathrm{lung}$ ). Allysine content (Figure $3 \mathrm{E}$ ) followed a trend similar to that of LOX activity and increased significantly at 1 week $(10.0 \pm 1.0 \mathrm{nmol} / \mathrm{lung}$ vs. sham: $3.8 \pm 1.5 \mathrm{nmol} / \mathrm{lung})$, continued to increase at 2 weeks (15.3 $\pm 1.7 \mathrm{nmol} / \mathrm{lung})$, and significantly decreased at 4 weeks $(8.5 \pm 3.9 \mathrm{nmol} / \mathrm{lung})$. Gd-Hyd-enhanced MRI similarly tracked allysine content at 2 and 4 weeks (Figure 3F), quantifiable by the significantly increased $\triangle \mathrm{CNR}$ at 2 weeks ( $4.9 \pm 2.6$ vs. sham: $0.2 \pm 0.4$; a 25 -fold increase), and significantly decreased $\triangle \mathrm{CNR}$ at 4 weeks ( $2.2 \pm 1.7$, a $55 \%$ reduction) (Figure $3 \mathrm{G}$ ).

Next we evaluated whether Gd-Hyd could be effective at detection and staging of hepatic fibrosis. Liver imaging was feasible given the rational design of Gd-Hyd to allow rapid probe clearance while minimizing nonspecific hepatic uptake. After carbon tetrachloride $\left(\mathrm{CCl}_{4}\right)$ treatment to induce liver fibrosis in mice, we first tested the specificity of Gd-Hyd by comparing it to Gd-DiMe in mice treated with $\mathrm{CCl}_{4}$ or vehicle for 12 weeks (Supplemental Figure 10). We imaged before and 15 minutes after bolus intravenous injection 

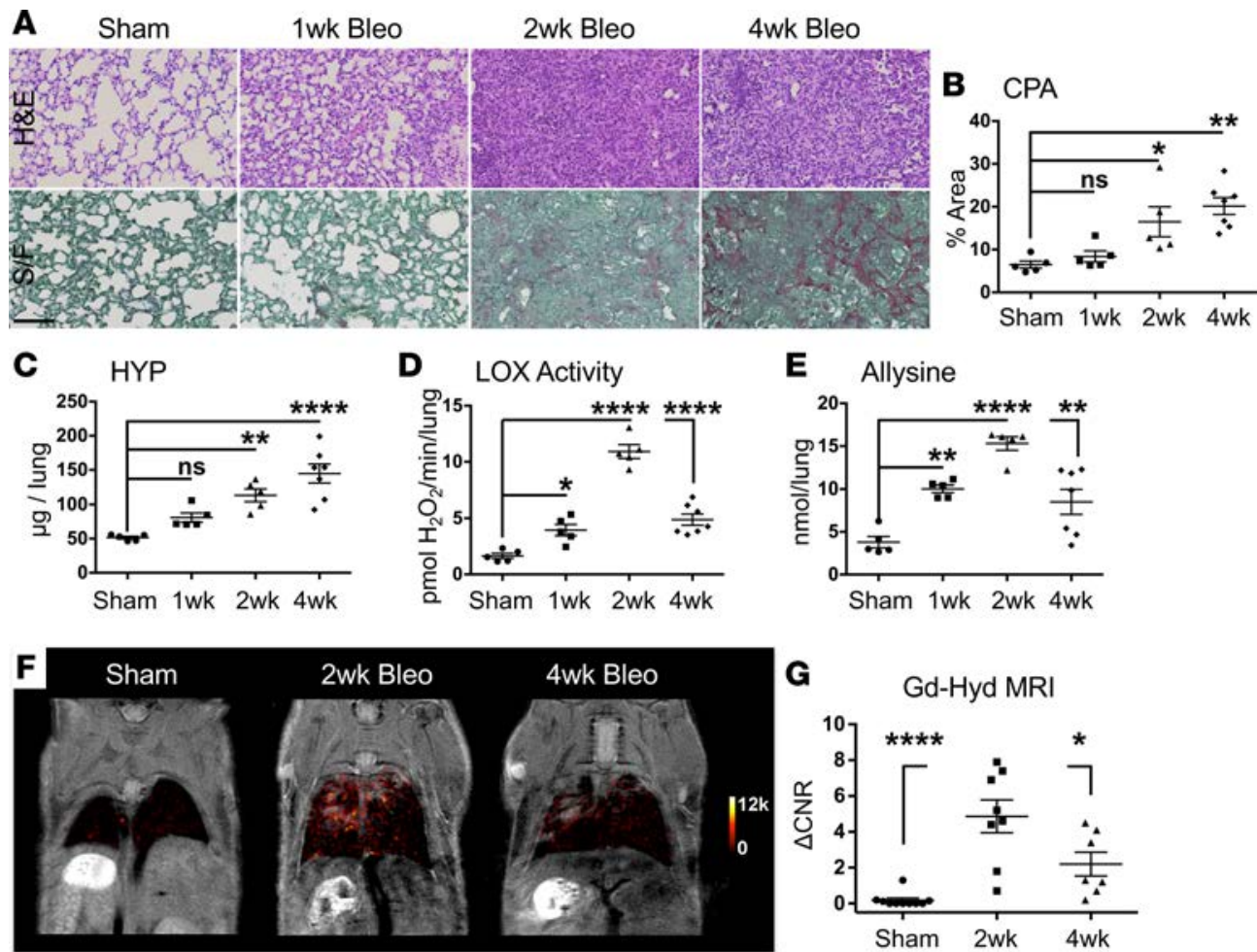

Figure 3. Gd-Hyd distinguishes active pulmonary fibrogenesis from stable scar. (A) To validate the pathogenesis in the lower-dose bleomycin model, $\mathrm{H} \& \mathrm{E}$ and Sirius Red/fast green (S/F) staining were performed in mice that received PBS (sham, $n=5$ ) or were challenged with 1 unit/kg bleomycin intratracheally 1 week (1wk, $n=$ 5), 2 weeks ( 2 wk, $n=5$ ), or 4 weeks prior ( $4 w k, n=7)$. Lung injury was assessed by Ashcroft score (see text), collagen proportional area (CPA) (B), lung hydroxyproline (HYP) content (C), lysyl oxidase (LOX) activity (D), and allysine content (E). (F) Gd-Hydenhanced coronal MR images showed minimal enhancement in the sham, robust enhancement at 2 weeks after bleomycin, and reduced enhancement at 4 weeks. (G) Change in contrast-tonoise ratio ( $\triangle \mathrm{CNR}$ ) quantification of Gd-Hyd. Scale bar: $100 \mu \mathrm{m} .{ }^{*} P<0.05$, ${ }^{* *} P<0.01,{ }^{* * *} P<0.0001$, ns, not significant; ANOVA.

of Gd-Hyd or Gd-DiMe at $100 \mathrm{nmol} / \mathrm{g}$. As in the lung we computed $\triangle \mathrm{CNR}$ for the change in liver versus muscle CNR following probe administration. Because of the fast clearance, Gd-Hyd showed essentially no liver enhancement in vehicle-treated mice but there was a strong, significant liver enhancement in the mice treated with $\mathrm{CCl}_{4}$ for 12 weeks. Gd-DiMe showed weak liver enhancement that was not different between the $\mathrm{CCl}_{4}$ - and vehicle-treated groups.

We then tested whether Gd-Hyd-enhanced MRI could detect liver fibrosis, monitor disease progression, and monitor disease regression induced by toxin withdrawal. Mice were treated with $\mathrm{CCl}_{4}$ or vehicle for 6 or 12 weeks, and another cohort of mice received $\mathrm{CCl}_{4}$ for 6 weeks followed by a 6 -week withdrawal period. After imaging, the livers were removed and analyzed. Hepatic fibrosis increased with the duration of $\mathrm{CCl}_{4}$ treatment, and resolved once the toxin was withdrawn, as assessed by Sirius Red staining (Figure 4A). A pathologist blinded to the study scored the sections for fibrosis using the Ishak scale (Figure 4B). Ishak score was significantly increased at 6 weeks $(3.2 \pm 1.5$, range $0-5)$, further increased at 12 weeks $(4.4$ \pm 1.0 , range $3-6$ vs. vehicle: $0.3 \pm 0.5$, range $0-1)$, and significantly decreased with $\mathrm{CCl}_{4}$ withdrawal $(1.4 \pm$ 0.8 , range $1-3)$. CPA in the livers (Figure $4 \mathrm{C})$ was significantly higher at 6 weeks $(2.7 \% \pm 0.8 \%$ vs. vehicle: $0.55 \% \pm 1.9 \%)$, further increased at 12 weeks $(4.0 \% \pm 1.2 \%)$, but significantly lowered after withdrawal $(1.5 \% \pm 0.2 \%)$. HYP content (Figure 4D) was nonsignificantly increased at 6 weeks $(315.7 \pm 128.8 \mu \mathrm{g} / \mathrm{g}$ liver vs. vehicle: $202.2 \pm 79.4 \mu \mathrm{g} / \mathrm{g}$ liver), significantly increased at 12 weeks (500.6 $\pm 158.6 \mu \mathrm{g} / \mathrm{g}$ liver), and significantly decreased in the withdrawal group $(259.6 \pm 105.2 \mu \mathrm{g} / \mathrm{g}$ liver $)$. These measures indicated increasing fibrosis with the duration of $\mathrm{CCl}_{4}$ treatment, while the withdrawal group showed significant decreases in each of these measures to values approaching those of the vehicle-treated controls. Analysis of mRNA levels of Lox and Lox-like genes (Figure 4, E and F, and Supplemental Figure 11) showed significantly increased expression for Loxl1 and Loxl2 in response to $\mathrm{CCl}_{4}$, but reduced to basal levels upon toxin withdrawal. Assays used to measure total LOX activity and allysine content could not be performed in the liver because of the high concentration of endogenous oxidative enzymes.

Representative images before and after Gd-Hyd injection show liver signal increases that correlate with disease severity (Figure 4, G and $\mathrm{H}$ ). $\triangle \mathrm{CNR}$ was significantly increased in mice treated with $\mathrm{CCl}_{4}$ for 6 weeks $(1.2 \pm 0.8$ vs. vehicle: $0.1 \pm 0.2$; a 15 -fold increase), and for 12 weeks $(2.0 \pm 1.3,24$-fold higher $)$, while in the $\mathrm{CCl}_{4}$-withdrawal group the $\triangle \mathrm{CNR}(0.5 \pm 0.9)$ was not significantly higher than the vehicle-treated controls (Figure 4I). The imaging data mirrored disease progression and resolution as assessed by Ishak scoring, 

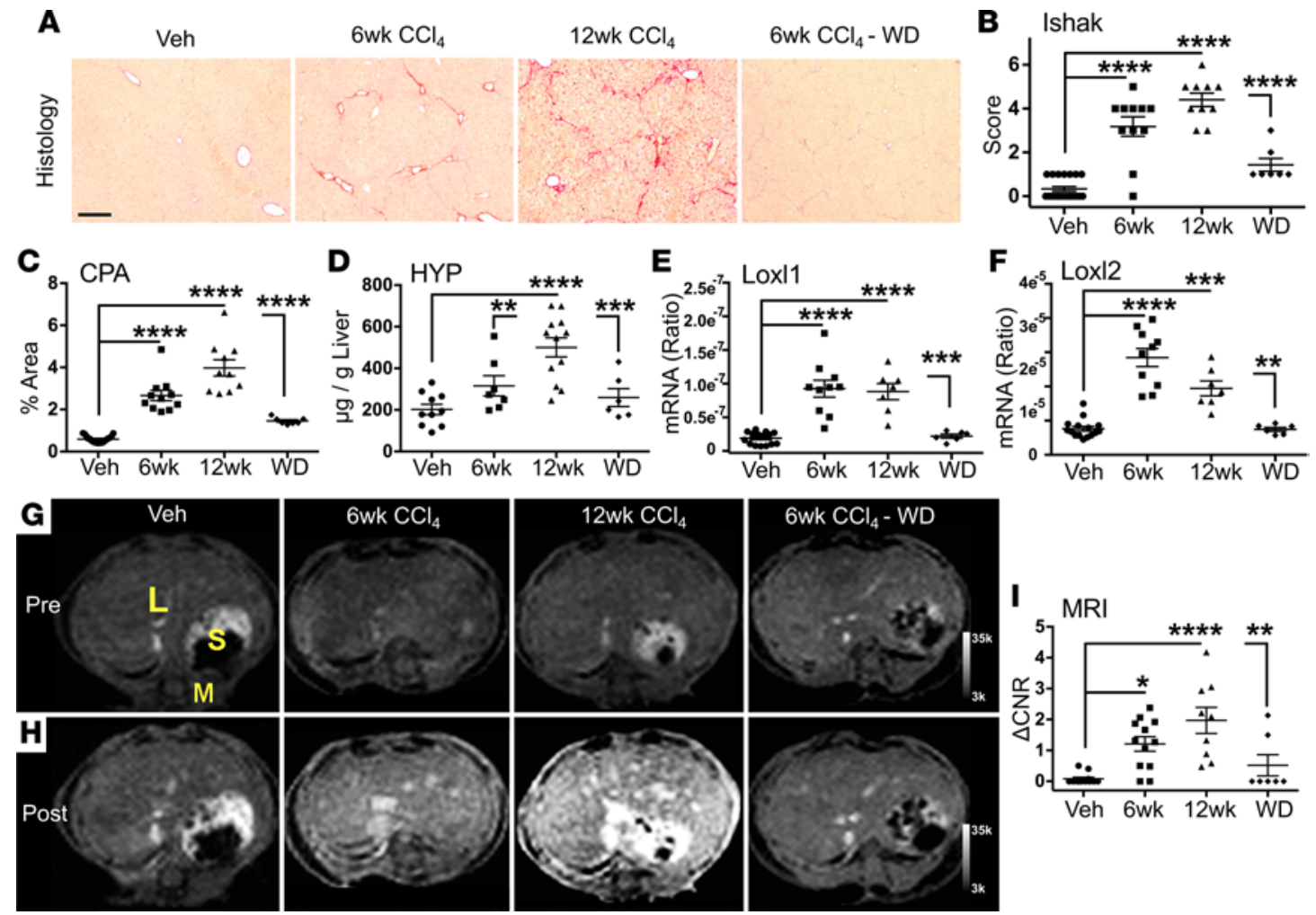

Figure 4. Gd-Hyd-enhanced MRI of hepatic fibrosis progression and regression. Mice were treated with vehicle $(n=12)$ or $C C C_{4}$ for 6 weeks $(n=12)$, for 12 weeks $(n=9)$, or for 6 weeks followed by 6 weeks of withdrawal (WD, $n=7$ ). Fibrosis in the liver was assessed by (A) Sirius Red staining, (B) Ishak scoring, (C) collagen proportional area (CPA), (D) hydroxyproline (HYP), and (E and F) Loxl1 and LoxI2 gene expression. (C) MRI without Gd-Hyd could not distinguish diseased mice. (H) After Gd-Hyd injection, liver enhancement was proportional to the extent of disease, quantified by the change in contrast-to-noise ratio $(\Delta \mathrm{CNR})(\mathrm{I})$. Scale bar: $100 \mu \mathrm{m}$. L, liver; S, stomach; M, muscle. ${ }^{*} P<0.05,{ }^{* *} P<0.01,{ }^{* * *} P<0.001,{ }^{* * *} P<0.0001 ;$ ANOVA.

CPA, HYP quantification, and Loxl1 and Loxl2 mRNA expression. Taken together, these results show that $\mathrm{Gd}-\mathrm{Hyd}$ noninvasively tracked disease progression and resolution in a $\mathrm{CCl}_{4}$-induced liver fibrosis model.

\section{Discussion}

Current noninvasive methods to assess tissue fibrosis and fibrogenesis are limited. High-resolution computed tomography (HRCT) is invaluable in diagnosing idiopathic pulmonary fibrosis and relies on specific patterns of opacification in the lungs. Increased fibrosis is indicated by increased areas and density of opacification. HRCT cannot distinguish active fibrogenesis from stable disease, however. Elastography methods using ultrasound or MR are increasingly used in the liver to detect fibrosis. These methods assess tissue stiffness, which increases with increasing fibrosis. These methods are weighted to more advanced disease, however, and lack sensitivity to the specific detection of active fibrogenesis.

We present here a fibrogenesis-sensing MRI contrast agent, Gd-Hyd, which targets the high micromolar concentration of allysine present during active fibrosis. Gd-Hyd enables noninvasive and direct investigation of the changes in the extracellular matrix microenvironment in vivo. The simple and scalable chemistry of Gd-Hyd, its immediate target localization after injection, its fast background washout, and the standard imaging protocols utilized here suggest a clinically translatable paradigm that is universal for monitoring of fibrotic disease progression and treatment response across organ systems.

During fibrogenesis, matrix proteins are overexpressed and LOX catalyzes oxidation of matrix (mostly collagen) lysine residues to the aldehyde allysine. These protein aldehydes then undergo noncatalytic condensation reactions to create stable crosslinks (see Supplemental Scheme 3). Matrix allysine represents an attractive molecular marker of fibrogenesis. In the absence of active disease, LOX family member expression levels drop and the allysine residues will eventually undergo condensation/crosslinking reactions and disappear. We directly quantified the amount of allysine present in lung tissue by HPLC. In the lung, allysine was elevated at 1 week after bleomycin challenge, reached significantly increased levels at 2 weeks after 
bleomycin, but was significantly reduced 4 weeks after bleomycin insult, at which point the injury became a stable fibroelastic scar $(29,30)$. Allysine production could be blocked by treating bleomycin-injured mice with the pan-LOX inhibitor BAPN. MRI with Gd-Hyd noninvasively tracked these changes in allysine and could distinguish active fibrogenesis occurring at 2 weeks after bleomycin from the stable scar present at 4 weeks after bleomycin. On the other hand, HYP, a common measure of total collagen, was not a good marker of fibrogenesis in this model, with similar levels observed at both 2 and 4 weeks after bleomycin. Of note, many antifibrotics currently being tested have been shown to reduce LOX family gene expression, and therefore Gd-Hyd could be an early, noninvasive measure of reduced fibrogenesis in clinical trials.

Among molecular imaging techniques currently being developed, MRI offers many benefits over nuclear imaging techniques: much higher spatial resolution, no ionizing radiation, excellent soft tissue contrast, and the ability to generate multiple image contrasts. The main limitation of molecular MRI is detection sensitivity, which is in the micromolar range for MRI probes. The allysine levels measured in fibrotic lung were greater than $100 \mu \mathrm{M}(100 \mathrm{nmol} / \mathrm{g})$, making allysine an attractive MRI target. Gd-Hyd was rationally designed to image fibrogenesis with an eye to clinical translation. The probe chemistry is simple, robust, and amenable to large-scale synthesis, utilizing a key intermediate that has already been manufactured for clinical development (31). The DOTA chelator used in Gd-Hyd is also used in the most stable commercial MRI contrast agent Gd-DOTA that has no reported cases of nephrogenic systemic fibrosis. GdHyd showed no release of Gd when challenged with zinc and phosphate under conditions where many approved MRI contrast agents will release Gd (Supplemental Figure 5), and was stable when incubated in serum for 24 hours (Supplemental Figure 6).

Gd-Hyd was designed to be small, anionic, and very hydrophilic to minimize nonspecific protein binding, to prevent cellular uptake, to enable rapid penetration into tissue interstitium, and to promote rapid renal elimination. When injected intravenously into the bloodstream, Gd-Hyd target localization is immediate with fast washout from nontarget organs, resulting in a strong target-to-background signal. The rapid uptake allows a baseline scan and a Gd-Hyd-enhanced scan to be acquired in the same imaging session, which is important for clinical workflow. The binding to allysine is reversible and overall elimination of gadolinium from the body is fast and complete with more than $99.5 \%$ cleared from the body after 24 hours (Supplemental Table 1). The rapid washout and subsequent clearance of Gd-Hyd also enables longitudinal serial imaging to track disease and treatment response. Here, we demonstrated repeated imaging feasibility in 2 instances: imaging the same 2-week post-bleomycin mouse with Gd-Hyd and Gd-DiMe on consecutive days, and imaging the same mouse at 2 and 4 weeks after bleomycin or PBS instillation. Furthermore, the imaging performed here utilized standard T1-weighted protocols that are available on commercial clinical scanners. Gd-Hyd imaging is thus well suited for translation into human patients to track disease progression and treatment response.

Gd-Hyd-specific detection of active fibrogenesis is complementary to state-of-the-art preclinical and clinical imaging methods to assess fibrosis. The spatial resolution of MRI is comparable to CT for both small animal and human imaging. While CT can stage pulmonary fibrosis, Gd-Hyd-enhanced MRI can report locally on regions of active fibrogenesis. In the liver, Gd-Hyd-enhanced MRI can be performed in the same study as MR elastography to provide complementary measures of fibrosis and fibrogenesis, as has been shown for other molecular imaging approaches (32).

There are some limitations to this report. The allysine target of Gd-Hyd is the product of LOX enzyme activity and the collagen substrate. We showed that Gd-Hyd-enhanced MRI detected fibrogenesis 1 week after bleomycin challenge when LOX activity was significantly elevated but HYP, a collagen surrogate, was not significantly increased over basal levels. However, we did not identify the earliest time point that Gd-Hyd could detect lung or liver injury. For simplicity we referred to LOX oxidation of collagen since this is the most abundant matrix protein, but other matrix proteins may be transformed. In the bleomycin model we showed that elastin is also increased with injury, although the highest elastin levels were observed 28 days after bleomycin when fibrosis is becoming more stable and Gd-Hyd imaging was decreased. In other diseases where elastogenesis is prominent, such as atherosclerosis or aneurysm, Gd-Hyd may also prove useful to assess disease activity.

In summary, the common collagen biology of fibrosis coupled with the low background signal from Gd-Hyd suggests that this probe would have broad applicability. In addition to the lung and liver studies shown here, Gd-Hyd-enhanced targeted molecular MRI would be able to detect and stage fibrosis in multiple other organs and tissues, including the heart in cardiomyopathy, myocardial infarction, and atrial 
fibrillation, the intestine in inflammatory bowel disease, the kidney in diabetic nephropathy, muscle in muscular dystrophy, arteries in atherosclerosis, and tumor stroma in cancers such as pancreatic cancer. Thus, Gd-Hyd should have broad applicability in the clinic where it could be used to identify patients with active fibrogenesis in a number of disease settings.

\section{Methods}

Full descriptions of materials and methods used are detailed in the supplemental material.

Molecular probe synthesis and characterization. Gd-Hyd was synthesized in a 3-step reaction designed based on the stable Gd-DOTA chelate. Gd-Hyd interaction with aldehyde was characterized in an in vitro binding assay with aldehyde-functionalized BSA, and with relaxivity measurements. Gd-Hyd binding to allysinerich porcine aorta tissue was further demonstrated. Gd-DiMe control probe was included in the assays to determine Gd-Hyd specificity. Probe pharmacokinetics in vivo and serum stability were also assessed.

MRI and quantification. All animal experiments were performed in compliance with institutional guidelines and were approved by the Institutional Animal Care and Use Committee at the Massachusetts General Hospital. A total of 87 C57BL6 mice were included in the pulmonary fibrosis study. Fiftytwo mice were intratracheally injected with 2.5 units of bleomycin per kg body weight, and imaged either (a) after 1 week when injury is mild $(n=9)$, (b) after 2 weeks when injury is severe $(n=16)$, (c) after daily intraperitoneal injection of LOX inhibitor BAPN $(n=12)$, or PBS $(n=15)$ for 2 weeks. Another 17 mice were injected with 1 unit of bleomycin per $\mathrm{kg}$ body weight and sacrificed at 1 week $(n$ $=5)$ or 2 weeks $(n=5)$, or serially imaged at 2 and 4 weeks and sacrificed $(n=7)$. Sham control mice were intratracheally injected with PBS $(n=18)$. 3D MR images were acquired on a 4.7 Tesla Bruker scanner with the ultrashort echo time sequence before and 12 minutes after bolus intravenous probe injection $(0.1 \mathrm{mmol} / \mathrm{kg}$ body weight).

A total of $66 \mathrm{~A} / \mathrm{J}$ mice were included in the hepatic fibrosis study. Liver fibrosis was induced by chronic oral gavage of $\mathrm{CCl}_{4}$ for 6 weeks $(n=19)$ or 12 weeks $(n=13)$. In a subset of animals, $\mathrm{CCl}_{4}$ was withdrawn after 6 weeks $(n=7)$ to allow fibrosis resolution. Sham control mice were fed vehicle only $(n=24)$. 3D FLASH images were acquired on a 4.7 Tesla Bruker scanner before and 15 minutes after bolus intravenous probe injection $(0.1 \mathrm{mmol} / \mathrm{kg}$ body weight).

Images were analyzed and quantified in Osirix by defining the region of interest that encompasses the diseased tissue, the muscle as control, and the background signal. CNR was determined by normalizing the probe enhancement in the diseased tissue to muscle. $\triangle \mathrm{CNR}$ was then computed by subtracting the CNR after probe injection from the CNR before probe injection. Statistical analysis was performed in GraphPad Prism.

Tissue histology and biochemical analysis. All animals were sacrificed after imaging and the diseased tissue was harvested. A portion was fixed and processed for H\&E staining, Sirius Red/fast green staining, Verhoeff's elastin staining, pathology scoring, and CPA quantification. The remaining fresh tissue was analyzed for gene expression changes, LOX activity, HYP measurements, and allysine levels.

Statistics. Statistical analyses were performed using GraphPad Prism. Results are expressed as the mean \pm 1 SEM unless otherwise noted. One-way ANOVA, followed by post-hoc Tukey tests with 2-tailed distribution was performed to analyze data among groups of 3 or more. Student's $t$ test compared data between control and 1 experimental group. $P$ less than 0.05 was considered significant.

\section{Author contributions}

HHC, PAW, LW, LFT, DTS, NJR, CMJ, RJL, GZ, JME, and CKP executed experiments, analyzed data, and prepared figures. HHC, PAW, and PC wrote the manuscript. HHC, PAW, AMT, KKT, ML, BCF, and PC designed the research study and edited the manuscript. LW, LFT, MM, and GYL analyzed histological data.

\section{Acknowledgments}

This work was supported by the following NIH grants: EB009062, DK104302, HL116315, HL131907 (to P.C.), and DK104956 (to B.C.F), and instrumentation grants S10OD010650 and S10RR023385.

Address correspondence to: Peter Caravan, 149 13th Street, Boston, Massachusetts 02129, USA. Phone: 617.643.0193; E-mail: caravan@nmr.mgh.harvard.edu. 
1. Friedman SL, Sheppard D, Duffield JS, Violette S. Therapy for fibrotic diseases: nearing the starting line. Sci Transl Med. 2013;5(167):167sr1.

2. Rockey DC, Bell PD, Hill JA. Fibrosis--a common pathway to organ injury and failure. N Engl J Med. 2015;372(12):1138-1149.

3. Ahluwalia N, Shea BS, Tager AM. New therapeutic targets in idiopathic pulmonary fibrosis. Aiming to rein in runaway woundhealing responses. Am J Respir Crit Care Med. 2014;190(8):867-878.

4. Blackwell TS, et al. Future directions in idiopathic pulmonary fibrosis research. An NHLBI workshop report. Am J Respir Crit Care Med. 2014;189(2):214-222.

5. Schuppan D, Afdhal NH. Liver cirrhosis. Lancet. 2008;371(9615):838-851.

6. Burstein B, Nattel S. Atrial fibrosis: mechanisms and clinical relevance in atrial fibrillation. J Am Coll Cardiol. 2008;51(8):802-809.

7. Jugdutt BI, Joljart MJ, Khan MI. Rate of collagen deposition during healing and ventricular remodeling after myocardial infarction in rat and dog models. Circulation. 1996;94(1):94-101.

8. Brilla CG, Funck RC, Rupp H. Lisinopril-mediated regression of myocardial fibrosis in patients with hypertensive heart disease. Circulation. 2000;102(12):1388-1393.

9. Sugimoto H, Grahovac G, Zeisberg M, Kalluri R. Renal fibrosis and glomerulosclerosis in a new mouse model of diabetic nephropathy and its regression by bone morphogenic protein-7 and advanced glycation end product inhibitors. Diabetes. 2007;56(7):1825-1833.

10. Mehal WZ, Iredale J, Friedman SL. Scraping fibrosis: expressway to the core of fibrosis. Nat Med. 2011;17(5):552-553.

11. Wynn TA. Cellular and molecular mechanisms of fibrosis. J Pathol. 2008;214(2):199-210.

12. Manning DS, Afdhal NH. Diagnosis and quantitation of fibrosis. Gastroenterology. 2008;134(6):1670-1681.

13. Popov Y, Schuppan D. Targeting liver fibrosis: strategies for development and validation of antifibrotic therapies. Hepatology. 2009;50(4):1294-1306.

14. Barry-Hamilton V, et al. Allosteric inhibition of lysyl oxidase-like-2 impedes the development of a pathologic microenvironment. Nat Med. 2010;16(9):1009-1017.

15. Rucker RB, et al. Modulation of lysyl oxidase by dietary copper in rats. J Nutr. 1996;126(1):51-60.

16. Caravan P, et al. Collagen-targeted MRI contrast agent for molecular imaging of fibrosis. Angew Chem Int Ed Engl. 2007;46(43):8171-8173.

17. Caravan $\mathrm{P}$, et al. Molecular magnetic resonance imaging of pulmonary fibrosis in mice. Am J Respir Cell Mol Biol. 2013;49(6):1120-1126.

18. Fuchs BC, et al. Molecular MRI of collagen to diagnose and stage liver fibrosis. J Hepatol. 2013;59(5):992-998.

19. Helm PA, et al. Postinfarction myocardial scarring in mice: molecular MR imaging with use of a collagen-targeting contrast agent. Radiology. 2008;247(3):788-796.

20. Polasek M, et al. Molecular MR imaging of liver fibrosis: a feasibility study using rat and mouse models. $J$ Hepatol. 2012;57(3):549-555.

21. Bornstein P, Kang AH, Piez KA. The nature and location of intramolecular cross-links in collagen. Proc Natl Acad Sci USA. 1966;55(2):417-424.

22. Pinnell SR, Martin GR. The cross-linking of collagen and elastin: enzymatic conversion of lysine in peptide linkage to alphaaminoadipic-delta-semialdehyde (allysine) by an extract from bone. Proc Natl Acad Sci USA. 1968;61(2):708-716.

23. Tanzer ML. Cross-linking of collagen. Science. 1973;180(4086):561-566.

24. Siegel RC. Biosynthesis of collagen crosslinks: increased activity of purified lysyl oxidase with reconstituted collagen fibrils. Proc Natl Acad Sci USA. 1974;71(12):4826-4830.

25. Port M, Idée JM, Medina C, Robic C, Sabatou M, Corot C. Efficiency, thermodynamic and kinetic stability of marketed gadolinium chelates and their possible clinical consequences: a critical review. Biometals. 2008;21(4):469-490.

26. Herborn CU, et al. Clinical safety and diagnostic value of the gadolinium chelate gadoterate meglumine (Gd-DOTA). Invest Radiol. 2007;42(1):58-62.

27. Tang SS, Trackman PC, Kagan HM. Reaction of aortic lysyl oxidase with beta-aminopropionitrile. J Biol Chem. 1983;258(7):4331-4338.

28. Ashcroft T, Simpson JM, Timbrell V. Simple method of estimating severity of pulmonary fibrosis on a numerical scale. J Clin Pathol. 1988;41(4):467-470.

29. Blaauboer ME, et al. Extracellular matrix proteins: a positive feedback loop in lung fibrosis? Matrix Biol. 2014;34:170-178

30. Riley DJ, et al. beta-Aminopropionitrile prevents bleomycin-induced pulmonary fibrosis in the hamster. Am Rev Respir Dis. 1982;125(1):67-73.

31. Levy SG, et al. Development of a multigram asymmetric Synthesis of 2-(R)-2-(4,7,10-Tris tert-butylcarboxymethyl-1,4,7,10tetraazacyclododec-1-yl)-pentanedioic acid, 1-tert-butyl ester, (R)-tert-Bu4-DOTAGA1. Org Process Res Dev. 2009;13(3):535-542.

32. Zhu B, et al. Combined magnetic resonance elastography and collagen molecular magnetic resonance imaging accurately stage liver fibrosis in a rat model. Hepatology. 2017;65(3):1015-1025. 